\title{
From the editor of the issue
}

Kate Dreyfus on a Jewish German jazz band exiled down under in 1939, portrayed in a documentary with some questionable features. Susanne Braun-Bau on the differences and commonalities of vocational training in Australia and Germany, with surprisingly good features identified in the former. Mandy Kretzschmar on how Australians changed their perceptions of Europeans after the 'Great War', with intriguing images culled from Australian journals such as The Bulletin. The racism and national prejudices which underpinned Australian political cartooning then is hard to believe. Post-structural Oliver Haag debating the truth/fiction binary in Aboriginal life writing and exposing astonishingly different perceptions between the writers and the critics of the genre. And finally Martin Grosch addressing a truly 'burning' issue for our times: how Sydney's urban sprawl is the main factor behind to the seemingly unstoppable rise in greenhouse gas emissions in Australia's five metropolitan cities (or "teeming sores" according to A.D. Hope.) But he also reports on some promising trends to revert the process. These are the five major essays in this new volume, but some of the reviews are hardly less than that. Consider Horst Prießnitz reviewing the New Cambridge History of Australian literature - he digs much deeper than your standard review would do and investigates the traditions of National literary histories as well as national Anthologies. "Never before" he boldly claims, "has a literary history been more willing to acknowledge Australia's embeddedness in overseas literary currents and socio-cultural developments." You will also enjoy Corinna Erckenbrechts review of an Outback thriller, one (the review not the thriller) that bubbles with topical issues and presents a wild menagerie of stock 'Aussie' characters:

cattle barons, magicians, prospectors, engineers, politicos, lawyers, game keepers, anthropologists, trackers, minors, mechanics, butchers, water-deviners, cops, water managers ...

and many more, more than can be mentioned here. Her enthusiasm with this tale is infectious. Or if you are more the academic type, then you will be delighted with Anja Schwarz's double review of Kate 
Grenville's two latest novels - and impressed with her critical acumen. Last but definitely not least I want to mention our multitalented transatlantic friend Mr Birns, who is giving us a bravura analysis of David Malouf's complex narrative art, exemplified by his approach to the Iliad, in Malouf's latest novel Ransom. As well, there is new poetry by Robyn Rowland, Diane Fahey, Beate Josephi and George Nicholson. In short, the contributors and I have endeavoured to put together another fat volume of learned, entertaining, sometimes provoking and polemical essays. Enjoy!

Klagenfurt, June 2010, Adi Wimmer

\section{Vorwort des Herausgebers dieser Nummer}

Kate Dreyfus schreibt über die "Syncopators", eine deutsch-jüdische Combo im australischen Kriegsexil. Susanne Braun-Bau erhellt Unterschiede im deutschen und australischen Berufsschulensystem und stellt überraschende Stärken des letzteren fest. Mandy Kretzschmar analysiert kollektive australische Sichtweisen auf Europa in der Zwischenkriegszeit und bezieht sich dabei auf interessantes Cartoon-Material. Oliver Haag brilliert mit einer poststrukturalistischen Analyse über den Wahrheitsgehalt von indigenen Autobiografien und konstatiert, dass die Autor/inn/en diesbezüglich ganz anders über ihre Texte denken als die Kritiker. Und schließlich legt Martin Grosch eine profunde, mit Tabellen und Karten untermauerte Studie über verkehrsgeografische Aspekte des Großraums Sydney vor. Die Fehler der Vergangenheit (Stichwort "urban sprawl"; A.D. Hope bezeichnete die fünf großen Metropolen Australiens als "teeming sores") rächen sich jetzt trotz allem Gegensteuern mit ständig ansteigendem $\mathrm{CO} 2$ Ausstoß in einem urbanen Großraum.

Das sind die fünf großen Beiträge dieses Bandes. Nicht weniger interessant sind eine Vielzahl von Rezensionen. Beachten Sie diesmal besonders den Review Essay von Horst Prießnitz über die 
New Cambridge History of Australian literature, in welcher nach Prießnitz im Vergleich früheren, mehr oder weniger nationalistisch eingefärbten Ansätzen eine Hinwendung zu internationalen Wurzeln und Einflüssen Platz greift. "Never before", so lautet sein Resumé, "has a literary history been more willing to acknowledge Australia's embeddedness in overseas literary currents and socio-cultural developments." Begeistert hat mich auch Corinna Erckenbrechts Besprechung eines "Outback Thriller", in dem eine pralle Handlung den Leser kaum zum Verschnaufen kommen lässt. Wer in Rezensionen den akademischen Duktus präferiert, wird bei Anja Schwarz und ihrer Doppelrezension zweier Romane von Kate Grenville gut aufgehoben sein. Eine beachtliche wissenschaftliche Leistung. Nicht zu vergessen auf unseren 'amerikanischen Freund' Nic Birns, der den neuesten Roman David Maloufs Ransom wortgewandt und kenntnisreich analysiert.

Daneben gibt es wiederum neue Lyrik von Robyn Rowland, Diane Fahey, Beate Josephi und George Nicholson. Kurz gesagt, die 26 Beiträger/inn/en und ich haben eine weiterer, substantielle Ausgabe der ZfA auf die Beine gestellt. Ich wünsche viel Lesegenuss!

Klagenfurt, Juni 2010, Adi Wimmer 